\title{
Diagnostische Fallstricke bei männlichem Patienten mit systemischem Lupus erythematodes
}

\author{
Andrei Cristian Dan Gheorghe ${ }^{a} \quad$ Andreea Simona Hodorogea $^{b} \quad$ Cristina Elena Georgescu ${ }^{c}$ \\ Ana Ciobanu $^{b}$ loan Tiberiu Naneab Gabriela Silvia Gheorghe ${ }^{b}$ \\ anternal Medicine, University of Medicine and Pharmacy Carol Davila, Prof. Dr. Th. Burghele Hospital, Bukarest, Rumänien; \\ ${ }^{b}$ Cardiology, University of Medicine and Pharmacy Carol Davila, Prof. Dr. Th. Burghele Hospital, Bukarest, Rumänien; \\ ${ }^{\complement}$ General Medicine, University of Medicine and Pharmacy Carol Davila, Sf. Pantelimon Clinical Emergency Hospital, Bukarest, Rumänien
}

\author{
Schlüsselwörter \\ Systemischer Lupus erythematodes · Antiphospholipid- \\ Antikörper · aPTT
}

\section{Zusammenfassung}

Systemischer Lupus erythematodes (SLE) ist eine chronische, multi-systemische, immunvermittelte Erkrankung mit irreführender Symptomatik und später Diagnosestellung. Wir berichten hier vom Fall eines 32-jährigen Mannes mit persistierender positiver VDRL-Reaktion (Venereal Disease Research Laboratory) nach Syphilistherapie 5 Jahre zuvor, der mit Exanthem, Gewichtsverlust, Panzytopenie, inflammatorischem Syndrom und erheblicher spontaner Verlängerung der aktivierten partiellen Thromboplastinzeit (aPTT) aufgenommen wurde. Bei dem Patienten wurden Antiphospholipid-Antikörper nachgewiesen und die Diagnose SLE gestellt. Das unerkannte falsch-positive VDRL-Ergebnis und die verzögerte SLE-Diagnose waren für den Patienten gesundheitsschädigend, da er zum Zeitpunkt der Diagnosestellung bereits renale und kardiale Komplikationen entwickelt hatte.

\section{Lerninhalte}

- Eine positive VDRL-Reaktion im Rahmen der Syphilis-Diagnostik muss durch andere Tests wie TPHA oder Western Blot gesichert werden, insbesondere wenn kein entsprechender klinischer Kontext vorliegt; die Möglichkeit eines falsch-positiven Ergebnisses muss bedacht werden.

- Eine spontane aPTT-Verlängerung kann mit dem Vorhandensein von Antiphospholipid-Antikörpern zusammenhängen.

- In der klinischen Praxis müssen Ärzte immer an eine Gesamtdiagnose für den jeweiligen Einzelfall denken, die alle klinischen Merkmale und laboranalytischen Daten einbezieht, auch wenn die Kombination zunächst unstimmig erscheint.

(c) EFIM 2019

\section{Beschreibung des Falls}

Ein 32-jähriger Mann wurde mit unbeabsichtigter Gewichtsabnahme (9 kg in 3 Monaten), makulopapulösem Exanthem an den Füßen, im Gesicht und im Brustbereich sowie morgendlicher Gelenksteifigkeit aufgenommen. Der Patient war Nichtraucher. In der Anamnese lagen vor: (a) erhöhter VDRL-Wert (Venereal Disease Research Laboratory), der 5 Jahre zuvor als Treponema-pallidum-Infektion interpretiert und mit Penicillin behandelt worden war; (b) eine moderate normochrome normozytäre Anämie, die 6 Monate vor der Aufnahme diagnostiziert und als Folge eines Vitaminmangels interpretiert worden war; sowie (c) ein inflammatorisches Syndrom, das als Folge einer 2 Monate vor der Aufnahme mit Ciprofloxacin behandelten Lungenentzündung interpretiert worden war.

Die körperliche Untersuchung ergab leichtes Fieber (37,5$38,0^{\circ} \mathrm{C}$ ), Hautblässe und ein nicht-juckendes, makulopapulöses Exanthem am Thorax (Abb. 1) und an den Fingerspitzen (Abb. 2). Es lagen keine pathologischen Veränderungen des kardiovaskulären, respiratorischen oder gastrointestinalen Systems vor. information@karger.com

www.karger.com/kai

Karger ${ }^{\prime \prime}=$
Andreea Simona Hodorogea

Cardiology

University of Medicine and Pharmacy Carol Davila, Prof. Dr. Th. Burghele Hospital Bukarest, Rumänien 


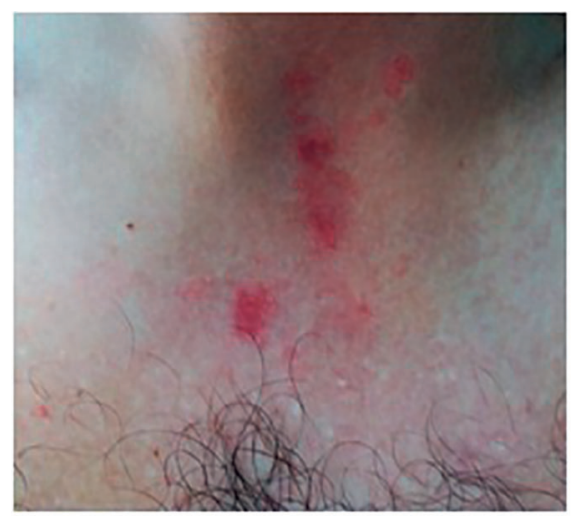

Abb. 1. Makulöse erythematöse Hautläsionen am Thorax.

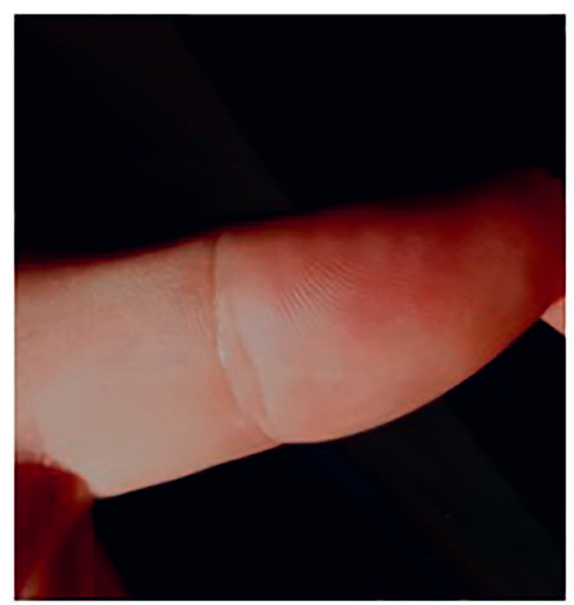

Abb. 2. Papulöser erythematöser Ausschlag an der Fingerkuppe.

Laboruntersuchungen ergaben Panzytopenie, einen niedrigen Eisengehalt im Blut, eine hohe Blutsenkungsgeschwindigkeit (BSG) und hohe Konzentration von C-reaktivem Protein, eine leicht herabgesetzte geschätzte glomeruläre Filtrationsrate, ein Albumin/ Kreatinin-Verhältnis von 100 mg/g im Urin, leicht erhöhte Kreatinphosphokinase- und Aspartat-Aminotransferase-Werte sowie okkulte gastrointestinale Blutungen. Der VDRL-Test erbrachte ein positives, der TPHA-Test (Treponema-pallidum-Hämagglutination) ein unklares und die Protein-Immunoblot-Reaktion (Western-Blot-Test) auf Treponema pallidum ein negatives Ergebnis. Gerinnungstests zeigten eine spontane erhebliche Verlängerung der aktivierten partiellen Thromboplastinzeit (aPTT) (Tab. 1). Der Patient hatte keine Gerinnungsstörungen in der Familien- oder persönlichen Anamnese, wendete keine Antikoagulanzien an und hatte keine Lebererkrankung. Wir testeten den Patienten auf Antiphospholipid-Antikörper (aPL) und erhielten für Lupus-Antikoagulans (LA) ein positives Ergebnis mit einem Verhältnis von 3,49, für Anticardiolipin-Ig-G-Antikörper (aCL) einen Wert von über $280 \mathrm{GPL}$ und für Antikörper gegen Beta2-Glykoprotein-IgG (aß2-GPI) ebenfalls ein positives Ergebnis. Solche aPL können zwar auch ohne identifizierbare Ursache auftreten, meist sind sie jedoch auf eine Primärerkrankung zurückzuführen. Anhand der Anamnese und des klinischen Zustands des Patienten wurde der Verdacht auf systemischen Lupus erythe- matodes (SLE) erhoben. Ein Test auf Antikörper gegen doppelsträngige DNA (dsDNA) war positiv (178 IU/ml) (Tab. 1).

Röntgen-Thorax, abdominale Sonographie und Endoskopie des oberen Gastrointestinaltrakts waren ohne Befund, während die Koloskopie blutende Hämorrhoiden ergab.

Wir gelangten zu dem Schluss, dass der Patient an SLE litt, da er mehrere der ACR/EULAR-Kriterien von 2018 erfüllte: Beteiligung des Muskel-Skelett-Systems, Leukopenie, Thrombozytopenie, Beteiligung der Nieren, aPL-positiv und dsDNA-Antikörperpositiv.

Im Laufe des Krankenhausaufenthalts traten Thoraxschmerz sowie erhöhte NT-proBNP- und Troponin-Serumkonzentrationen auf. Eine Kardio-MRT ergab das Bild einer Lupus-Myokarditis.

Nach 3-monatiger Behandlung mit $32 \mathrm{mg}$ Methylprednisolon und $200 \mathrm{mg}$ Hydroxychloroquin täglich normalisierten sich die Gerinnungswerte und das Blutbild des Patienten, die Proteinurie klang ab, und auch die NT-proBNP-Konzentration normalisierte sich.

\section{Diskussion}

Der Fall eines jungen Mannes mit langjähriger Vorgeschichte einer positiven VDRL-Reaktion, kürzlich aufgetretenem Hautausschlag und leichtem Fieber wirft bei der Differenzialdiagnose vielschichtige Probleme auf. Der erste Verdacht fällt auf eine sekundäre Syphilis, doch eine falsch-positive VDRL-Reaktion kann auch andere Ursachen haben, unter anderem eine akute Infektionskrankheit (Tab. 2) [1, 2]. Das klinische Bild unseres Patienten deckte sich mit keiner dieser Diagnosen. Der VDRL-Test ist ein nicht-Treponema-spezifisches Syphilis-Screening; die Diagnose muss gegebenenfalls durch einen Treponema-Test wie TPHA gesichert werden. Die TPHA-Reaktion kann jedoch bei infektiöser Mononukleose unklar und bei SLE falsch-positiv ausfallen [1]. Der nächste Schritt zur Diagnosesicherung ist ein Western-Blotting-Test oder eine Polymerase-Kettenreaktion [1]. Bei diesem Patienten fiel der TPHA-Test unklar aus, ein Western-Blot-Test auf Treponema pallidum jedoch negativ, sodass eine Syphilis ausgeschlossen werden konnte. Die Kombination aus falsch-positiven VDRL- und TPHA-Ergebnissen, Exanthem, Fieber, Inflammation, Panzytopenie und renaler Beteiligung kommt häufig bei SLE vor, insbesondere bei Frauen. Für diese Diagnose sprach auch die Bestätigung eines Antiphospholipid-Syndroms, auf welches die spontane sehr ausgeprägte aPTT-Verlängerung hingedeutet hatte.

Eine verlängerte aPTT kann auch andere Ursachen haben (Tab. $3)$, von denen in diesem Fall jedoch keine vorlag. Bei etwa 20 40\% der Patienten mit SLE liegen Antiphospholipid-Antikörper vor (aCL, anti-ß2-GPI, LA). Bei der Hälfte davon fällt auch der VDRL-Test positiv aus, wie es auch bei unserem Patienten der Fall war. Es besteht ein hohes Risiko für (häufig rezidivierende) tiefe Venenthrombosen, arterielle Thrombosen und Lungenembolien, insbesondere bei LA-positiven Patienten im Vergleich zu Patienten mit aCL-Antikörpern. In einer Studie von Pengo et al. bei 27 Patienten mit SLE war das gleichzeitige Vorliegen von LA, aCL 
Tabelle 1. Laborwerte

\begin{tabular}{|c|c|c|}
\hline Test & Wert des Patienten & Normwert/-bereich \\
\hline Erythrozyten $(/ \mu \mathrm{l})$ & $3,6 \times 103$ & $3,8-6,5 \times 103$ \\
\hline $\mathrm{Hb}(\mathrm{g} / \mathrm{dl})$ & 9,7 & $11,5-17,0$ \\
\hline$\overline{\mathrm{Ht}}(\%)$ & 30,7 & $37-54$ \\
\hline$\overline{\mathrm{MCV}}(\mathrm{fl})$ & 85,7 & $80-100$ \\
\hline$\overline{\mathrm{MCH}}(\mathrm{pg})$ & 27,1 & $27-32$ \\
\hline Leukozyten $(/ \mu \mathrm{l})$ & $3,8 \times 103$ & $4,0-10,0 \times 103$ \\
\hline Thrombozyten $(/ \mu \mathrm{l})$ & $135 \times 103$ & $150-450 \times 103$ \\
\hline Serum-Eisengehalt ( $\mu \mathrm{g} / \mathrm{dl})$ & 42 & $60-170$ \\
\hline Vitamin B12 (pg/ml) & 250 & $197-771$ \\
\hline Folsäure (ng/ml) & 26 & $4-34$ \\
\hline Peripherer Blutausstrich & \multicolumn{2}{|c|}{$\begin{array}{l}\text { Moderate Panzytopenie mit Erythrozytopenie mit ausgeprägter } \\
\text { Anisozytose (Mikrozytose mit Makrozytose), moderate Hypochromie, } \\
\text { Poikilozytose (Echinozyten, Schistozyten, Ovalozyten), Leukopenie } \\
\text { mit Neutropenie, Thrombozytopenie, Thrombozyten-Anisozytose mit } \\
\text { Makrothrombozyten }\end{array}$} \\
\hline Gastrointestinale Blutung & positiv & negativ \\
\hline$\overline{\mathrm{BSG}}(\mathrm{mm} / 1 \mathrm{~h})$ & 88 & $2-15$ \\
\hline Fibrinogen (mg/dl) & $1.003,71$ & $190-450$ \\
\hline$\overline{\text { C-reaktives Protein }(\mathrm{mg} / \mathrm{dl})}$ & 49,64 & $0-5$ \\
\hline Ferritin (ng/ml) & 604 & $21,81-274,66$ \\
\hline Procalcitonin (mg/l) & 0,2 & $0-5$ \\
\hline Serumalbumin $(\mathrm{g} / \mathrm{dl})$ & 43,4 & $3,5-5,5$ \\
\hline Kreatinin $(\mathrm{mg} / \mathrm{dl})$ & 1,21 & $0,7-1,3$ \\
\hline $\operatorname{eGFR}\left(\mathrm{ml} / \mathrm{min} / 1,72 \mathrm{~m}^{2}\right)$ & 73,3 & $\geq 90$ \\
\hline Glykämie (mg/dl) & 80 & $70-110$ \\
\hline$\overline{\mathrm{AST}}(\mathrm{IU} / \mathrm{I})$ & 42,73 & $1-37$ \\
\hline$\overline{\mathrm{ALT}}(\mathrm{IU} / \mathrm{I})$ & 30 & $1-41$ \\
\hline Gesamtbilirubin (mg/dl) & 0,26 & $0,2-1,2$ \\
\hline Alkalische Phosphatase (IU/I) & 76 & $30-129$ \\
\hline Antikörper gegen HCV & liegen nicht vor & liegen nicht vor \\
\hline $\mathrm{Hbs}-\mathrm{Ag}$ & negativ & negativ \\
\hline Antikörper gegen HIV 1 und 2 & liegen nicht vor & liegen nicht vor \\
\hline Kreatinphosphokinase (IU/I) & 191 & $38-171$ \\
\hline Troponin I (ng/dl) & 0,2 & $<1$ \\
\hline$\overline{\mathrm{TSH}}(\mu \mathrm{l} \mathrm{U} / \mathrm{ml})$ & 0,6 & $0,27-4,2$ \\
\hline Freies T4 (ng/dl) & 1,05 & $0,58-1,64$ \\
\hline $\mathrm{aTPO}(\mathrm{IU} / \mathrm{ml})$ & 2 & $0-9$ \\
\hline Antistreptolysin O (IU/I) & $<200$ & $<200$ \\
\hline$\overline{\mathrm{TPHA}}$ & unklar & negativ \\
\hline$\overline{\text { VDRL }}$ & positiv & negativ \\
\hline Western-Blot-Test auf Treponema pallidum & negativ & negativ \\
\hline Bakterielle Urinanalyse & steril & steril \\
\hline Urinanalyse: Proteinurie (mg/dl) & 50 & liegt nicht vor \\
\hline Albumin/Kreatinin-Verhältnis im Urin (mg/g) & 100 & $0-30$ \\
\hline PT-Zeit (s) & 18,2 & $10-15,1$ \\
\hline Prothrombin-Aktivität (\%) & 58,14 & $70-140$ \\
\hline$\overline{\mathrm{INR}}$ & 1,39 & $0,8-1,16$ \\
\hline$\overline{\mathrm{aPTT}}(\mathrm{s})$ & über der Nachweisgrenze & $<40$ \\
\hline
\end{tabular}

ALT: Alanin-Transaminase; AST: Aspartat-Transaminase; aPTT: aktivierte partielle Thromboplastinzeit; aTP: Antikörper gegen thyreoidale Peroxidase; eGFR: geschätzte glomeruläre Filtrationsrate; BSG: Blutsenkungsgeschwindigkeit; T4: Thyroxin; Hb: Hämoglobin; Ht: Hämatokrit; INR: Internationale Normalisierte Ratio; MCH: mittleres korpuskuläres Hämoglobin; MCV: mittleres korpuskuläres Volumen; MPV: mittleres Thrombozytenvolumen; RDW: Erythrozyten-Verteilungsbreite; TPHA: Treponema-pallidum-Hämagglutinationstest; TSH: thyreotropes Hormon; VDRL: Venereal Disease Research Laboratory.

und anti- $\beta 2$-GPI ein unabhängiger Risikofaktor für thrombotische Ereignisse [3].

Gemäß den Sapporo-Kriterien gehört zum AntiphospholipidSyndrom auch das Vorliegen einer Thrombose. Wenn keine Thrombose vorliegt, ist der Patient ein Träger von Antiphospho-
lipid-Antikörpern und unterliegt einem erhöhten Risiko für künftige Thrombosen [4].

Im weiteren Verlauf trat bei unserem Patienten eine Myokarditis auf. Dies kommt in Verbindung mit SLE seltener vor als Perikarditis oder Koronararteriitis. Die klinische Prävalenz der Lupus- 
Tabelle 2. Mögliche Ursachen einer falsch-positiven VDRL-Reaktion [1, 2]

\begin{tabular}{ll}
\hline Akutes Setting & Chronisches Setting \\
\hline Endokarditis & Systemischer Lupus erythematodes \\
Rickettsieninfektion & Thyroiditis \\
Infektiöse Mononukleose & Tuberkulose \\
Virale Pneumonie & Colitis ulcerosa \\
Varizellen & Vaskulitis \\
Masern & Rheumatoide Arthritis \\
Impfungen & Polyarteriitis nodosa \\
Akute Virushepatitis & Lepra \\
Brucellose & Höheres Alter \\
\hline
\end{tabular}

Tabelle 3. Mögliche Ursachen einer aPTT-Verlängerung [3]

Ursachen der aPTT-Verlängerung

Faktor-VIII-Wirkungsgrad (Hämophilie A)

Faktor-IX-Mangel (Hämophilie B)

Faktor-XI-Mangel

Erworbener Faktor-VIII-Inhibitor

Von-Willebrand-Syndrom

Antiphospholipid-Syndrom

Behandlung mit unfraktioniertem Heparin

Leberkrankheit

Dysfibrinogenämie

Spezifische Faktor-V-Inhibitoren

Myokarditis beträgt 9\%, allerdings haben Post-mortem-Untersuchungen gezeigt, dass sie in $57 \%$ der Fälle vorhanden ist. Die myokardiale Beteiligung bei SLE ist oft fokal ausgeprägt und dadurch mittels Endomyokardbiopsie schwer zu beurteilen [5]. Bei unserem Patienten lieferten die klinischen Daten, die erhöhten Tropo- nin- und NT-proBNP-Werte sowie die Kardio-MRT Hinweise auf die Diagnose. Die kardiale Beteiligung wurde durch die SLE-Medikamente plus eine unterstützende Behandlung der Herzinsuffizienz erfolgreich behandelt.

\section{Schlussfolgerungen}

Multisystemische, langfristige Symptome müssen hier in eine Gesamtdiagnose integriert werden. Ein positiver nicht-Treponemaspezifischer VDRL-Test, der auf die Diagnose Syphilis hindeutet, muss durch andere, Treponema-spezifische Tests bestätigt werden, insbesondere wenn kein entsprechender klinischer Kontext vorliegt. Bei unserem Patienten war der TPHA-Test unklar, der Western-Blot-Test auf Syphilis jedoch negativ. Die spontane starke aPTT-Verlängerung bei einem jungen Patienten mit verschiedenen Langzeitsymptomen erweckte den Verdacht auf ein Antiphospholipid-Syndrom im Rahmen des SLE. Bei unserem Patienten erklärte die lange Krankheitsdauer die zum Zeitpunkt der SLE-Diagnose bereits vorhandenen renalen und myokardialen Komplikationen.

\section{Interessenkonflikte}

Die Autoren erklären, dass keine Interessenkonflikte bestehen.

\section{Lizenzangabe}

Andrei Cristian Dan Gheorghe, Andreea Simona Hodorogea, Cristina Elena Georgescu, Ana Ciobanu, Ioan Tiberiu Nanea, Gabriela Silvia Gheorghe: Diagnostic pitfalls in a man with systemic lupus erythematous. Eur J Case Rep Intern Med. 2019;6:001256 (https://doi.org/10.12890/2019_001256), ( 2019 EFIM (Übersetzung), lizensiert unter CC BY 4.0 (https://creativecommons. org/licenses/by/4.0/deed/de).

\section{Literatur}

1 Larsen SA, Steiner BM, Rudolph AH: Laboratory diagnosis and interpretation of tests for syphilis. Clin Microbiol Rev. 1995;8:1-21.

2 Ratnam S: The laboratory diagnosis of syphilis. Can J Infect Dis Med Microbiol. 2005;16: 45-51.
Pengo V, Biasiolo A, Pegoraro C, et al.: Antibody profiles for the diagnosis of antiphospholipid syndrome. Thromb Haemost 2005;93: $1-6$.

4 Giannakopoulos B, Krilis SA: The pathogenesis of the antiphospholipid syndrome. N Engl J Med. 2013;368:1033-1044.
Tariq S, Garg A, Gass A, et al.: Myocarditis due to systemic lupus erythematosus associated with cardiogenic shock. Arch Med Sci 2018;14: 460-462. 